Thorax (1961), 16, 36.

\title{
THE LOWER END OF THE OESOPHAGUS
}

BY

\author{
JOHN HAYWARD \\ From the Royal Melbourne Hospital, Australia
}

(RECEIVED FOR PUBLICATION JULy 6, 1960)

The lower end of the oesophagus is a region where the pathology, the physiology, and even the anatomy are not quite clear. This paper attempts to dispel some of the confusion.

\section{Where is the Oesophago-gastric Junction ?}

The oesophagus or gullet is the tube which conducts food from the throat to the stomach, and the stomach is the pouch in which food collects and undergoes the initial stages of digestion and absorption. These simple definitions may seem so obvious as to be hardly worthy of mention, but they are fundamental.

From the outside it is easy to see where the tube ends and the pouch begins. As well as the abrupt change in shape there is the peritoneal reflection from stomach to diaphragm to mark the junction. It is therefore a definite, easily identified, anatomical region and will be referred to in this paper as the oesophago-gastric junction.

From the inside the only line of demarcation apparent is the wavy line of junction of stratified squamous with columnar epithelium; but it is not at the level of the oesophago-gastric junction as defined above. It is 1 or $2 \mathrm{~cm}$. higher. In longitudinal sections there is no sudden change in the macroscopic or microscopic appearance of the muscle coat or the submucosa at the oesophagogastric junction, and the columnar epithelium also passes without change across the junction into the stomach for a centimetre or two before it gradually alters to gastric epithelium of fundal type. Under the microscope the oesophago-gastric junction cannot be identified, so it is not surprising that histologists adopted the only line of demarcation available to them, namely the sudden change from squamous to columnar epithelium, and called the squamous epithelium oesophageal and the columnar epithelium gastric. Hence we have all been taught that the oesophagus is a tube lined by squamous epithelium.

This has put us in the ridiculous position of having the oesophago-gastric junction at a different level according to whether the tube is viewed from inside or outside. I firmly believe that this anomaly should be corrected and that the only way to correct it is to go back to the simple definition given above. The oesophagus is a tube and all of this tube is oesophagus, regardless of its lining. The true junction is where the conducting tube changes to the digesting pouch. This means that the oesophagus is not lined exclusively by squamous epithelium. Its lower 1 to $2 \mathrm{~cm}$. is lined by columnar epithelium, which also extends a little way into the stomach.

This columnar epithelium forms simple tubular glands, confined to the mucosa. It appears to produce a mucous secretion containing no digestive juices, but it obviously shares with fundal and pyloric epithelium the ability to resist acid-peptic digestion, because part of it is always in the stomach. The mucosa lined by it is very mobile. It normally moves up and down for a distance of nearly $2 \mathrm{~cm}$. As each bolus of food passes from oesophagus to stomach it pouts into the stomach and then retracts back again (Botha, 1958 ; Lerche, 1950). An important valvular action has been attributed to the pouting folds. Its movement results in continual variations of the portions of oesophagus and stomach lined by it. Such a mobile zone of epithelium does not make a satisfactory marker. It is confusing to give the area it covers a name, because in different phases of its normal function it covers different areas. It is better to give the epithelium itself a name, and to distinguish the lower part of the oesophagus by another name, and criteria other than its epithelial lining.

This epithelium has always been regarded as gastric in type and has been called cardiac epithelium, a rather absurd term which is saved from ambiguity because there happens to be no epithelium in the heart. Since, as will be shown below, it does not correspond to the cardia, I suggest that it be renamed "junctional epithelium."

The next point to be settled is whether it should continue to be regarded as gastric in type. In 
addition to the functions, possibly valvular, conferred by its mobility, it protects the squamous epithelium which is more susceptible to acid-peptic digestion. I suggest that if squamous epithelium joined gastric epithelium of fundal type directly, it would be liable to digestion at the junction. The buffer zone of junctional epithelium, which does not secrete acid or pepsin but is resistant to them, has to be interposed. It is easy to see why it must lie, as it does, astride the gastro-oesophageal junction partly in the oesophagus and partly in the stomach, for, if it were wholly in the oesophagus, fundal epithelium at the oesophago-gastric junction might secrete a little into the oesophagus, and if it were wholly in the stomach squamous epithelium at the oesophago-gastric junction would be in danger of digestion.

Junctional epithelium does not resemble fundal or pyloric epithelium. It is as dissimilar from them as colonic epithelium. The only characteristics shared by these four types of epithelium are that they are all columnar and all form glands. The function of the stomach is digestion and assimilation. Nobody has suggested that "cardiac" epithelium plays any part in digestion and it is doubtful if it assimilates. It resides as much in the oesophagus as in the stomach, and its movements and secretions are concerned entirely with oesophageal integrity and function. Similar epithelium also occurs normally near the upper end of the oesophagus. I suggest that junctional epithelium should be regarded as oesophageal.

Thus, the oesophageal lining should be described as having mainly stratified squamous epithelium with glands in the submucosa, but partly columnar epithelium with glands in the mucosa, in variable small islands near the upper end and round the whole circumference in the lower one or two centimetres. The stomach should be described as lined by two sorts of epithelium, fundal and pyloric, except for a small area round the oesophageal opening where oesophageal junctional epithelium protrudes into it.

\section{What is the Cardia?}

I have never seen or heard the cardia clearly defined. Is it just the gastro-oesophageal junction? The sphincter at this junction is called the constrictor cardiae. Is it the continually varying portions of lower oesophagus and upper stomach lined by junctional epithelium ? In that case it is only a general region with no fixed position. Is it the part which is narrow in the disease called achalasia of the cardia or cardiospasm ? If so, it is the lower $2 \mathrm{~cm}$. or so of the oesophagus, rather more than the junctional epithelium usually covers, and does not include the portion of the stomach lined by this epithelium. On the other hand, is it merely gastric and composed only of that portion of the stomach immediately surrounding the gastro-oesophageal junction, excluding the part of the oesophagus lined by junctional epithelium ?

The literature does not answer these questions. The cardia is often referred to as if it were part of the stomach, and a malignant growth arising in the junctional epithelium is called a carcinoma of the cardia and always regarded as a gastric neoplasm. At the same time the narrow zone in achalasia of the cardia is accepted as lower oesophagus and the inconsistency is conveniently ignored. I do not know exactly what the cardia is. The word does not appear in the index or in the text in Cunningham's (1951) or Gray's (Johnston, Davies, and Davies, 1958) textbooks or in Anatomy of the Human Body by Lockhart, Hamilton, and Fyfe (1959). These books describe the "cardiac orifice" as the opening of the oesophagus into the stomach and refer, vaguely as regards their position, to the " cardiac glands." Cunningham's textbook also states that the cardiac part of the stomach "comprises the fundus and the body of the stomach."

On the other hand, clinicians frequently use the word cardia and usually seem to mean by it the lower part of the oesophagus. Most of them indicate or imply that its lower limit is the oesophago-gastric junction, but none defines its precise upper limit. Hurst, in his now classical papers on achalasia of the cardia, never actually defined the cardia ; but he made it quite clear that the area concerned was the lower part of the oesophagus, and, writing with Rake (Hurst and Rake, 1930), stated that "we have come definitely to the conclusion that the last inch or more of the oesophagus has such a totally different function from that of the rest that it deserves to be regarded as a functional sphincter." This "last inch or more" was the nearest he ever got to defining the limits of what he implied was the cardia. To choose a recent example of the use of the word, Ellis, Kauntze, and Trounce (1960), describing experiments with pieces of living oesophageal muscle, stated that "at thoracotomy strips of normal oesophageal muscle were removed from the extreme lower end of the oesophagus (the cardia) ..." Again, the upper limit of the cardia is left unspecified. Their experiments showed that "the circular muscle of the cardia has a distinct pattern of innervation." In my opinion the length of the sphincteric part of the lower oesophagus approximates more to $2 \mathrm{~cm}$., but I am 
well aware of the difficulty of precise measurements in this region.

Since it is wise not to continue using a word which cannot be clearly defined, two possible courses are open: either to drop the word altogether, or to find some precise meaning for it. I propose the second.

Lerche (1950) reviewed the literature and made a study of the oesophageal musculature. He avoided the word cardia and called the lower $2 \mathrm{~cm}$. of the oesophagus the gastro-oesophageal vestibule, and described it as bounded above by the inferior oesophageal sphincter, indicated on the outside by the insertion of the phreno-oesophageal ligament, which is at the same level, and below by the constrictor cardiae at the oesophago-gastric junction. He paid little attention to the mucosa and submucosa which he removed from his specimens in order to show the muscle bundles from the inside.

There can be little doubt that sphincteric action occurs in the lower $2 \mathrm{~cm}$. of the oesophagus and that it is greater at the upper and lower ends of this part and less in the middle. When barium is watched on the fluorescent screen passing down the normal oesophagus there is a momentary holdup just above the diaphragm before the lower oesophagus opens and lets it through. During the hold-up the oesophagus above the sphincter may bulge considerably, producing the appearance known as phrenic (or, better, epiphrenic) ampulla. Also, when attempts are made to produce reflux of barium from a normal stomach, no reflux occurs and the barium stays in the stomach below the diaphragm. In the first example it is the inferior oesophageal sphincter situated near the upper surface of the diaphragm which is producing the hold-up. In the second it is the constrictor cardiae, probably aided by mucosal folds and other factors, situated below the diaphragm which is preventing the reflux.

These observations indicate the upper and lower limits of the gastro-oesophageal vestibule which lies partly within the oesophageal hiatus and partly below the diaphragm. As its upper part is encircled by the hiatal muscle it has been maintained that much of the apparent sphincteric action may be due to the diaphragmatic " pinchcock." This probably aids in the prevention of reflux at times when the diaphragm and abdominal wall muscles are contracted and the intra-abdominal pressure raised, but it is certain that intrinsic sphincters are present as well, in spite of the fact that they are not evident as thickenings of the circular muscle layer.

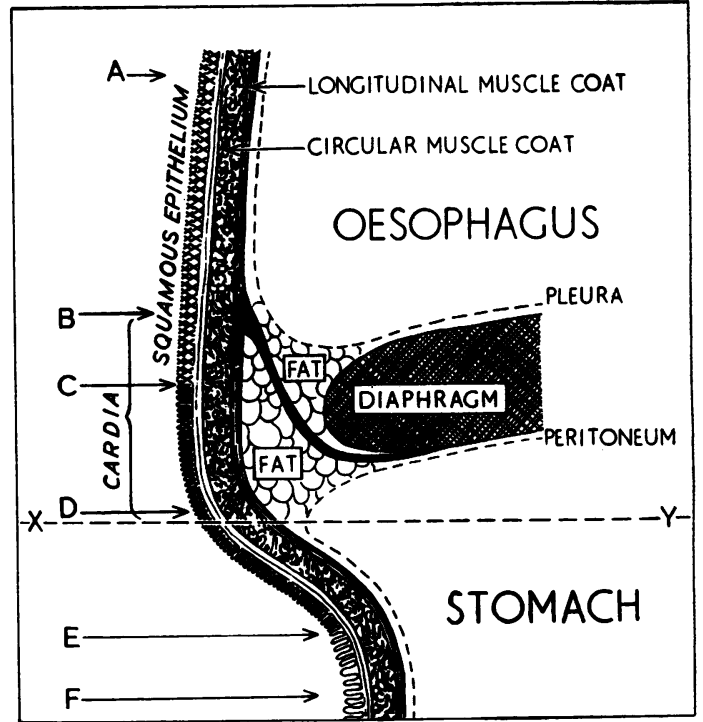

FIG. 1.-This diagram shows my conception of the anatomy of the oesophago-gastric junction and the terms which should be used in relation to it. Line XY crosses the oesophago-gastric junction. Everything above this line is oesophagus and everything below is stomach. A to $\mathbf{C}$ is lined by squamous epithelium. $B$ is the level of insertion of the phreno-oesophageal ligament. $B$ to $D$ is the cardia, i.e., the functioning muscular sphincter. $\mathbf{C}$ to $\mathbf{E}$ is the junctional epithelium. $\mathbf{F}$ is lined by funda epithelium. Thus the cardia is partially lined by squamous and partly by columnar epithelium and the junctional epithelium extends across the oesophago-gastric junction.

The part which refuses to open properly in cardiospasm is the lower $2 \mathrm{~cm}$., approximately, of the oesophagus. This part corresponds to the gastro-oesophageal vestibule described by Lerche. I cannot imagine the convenient term cardiospasm being replaced by " gastro-oesophageal vestibular spasm " or, if preferred, " achalasia of the gastrooesophageal vestibule." On the other hand, this part of the oesophagus is sufficiently distinct functionally to deserve a name of its own. I suggest that it be called the "cardia." This makes sense of the term " achalasia of the cardia" and makes the phrase "incompetence of the cardia" (in cases of sliding hiatal hernia) precise and correct. It is also easy to define. It is the sphincteric lower part of the oesophagus between the insertion of the phreno-oesophageal ligament and the gastrooesophageal junction. As the line of junction of the squamous and junctional epithelium is usually a little below the level of insertion of the phrenooesophageal ligament, the cardia, as defined, is normally lined mostly by junctional epithelium with a small, variable amount of squamous epithelium at the top. 
Regarding the cardia as the lower, sphincteric part of the oesophagus is in line with terminology elsewhere. The lower sphincteric part of the stomach, the pylorus, is not regarded as part of the duodenum, nor the ileo-caecal sphincter as part of the caecum. The anatomists' term, "cardiac orifice," for the opening of the oesophagus into the stomach, can remain unchanged.

\section{Pathological Implications}

If these opinions about the lower end of the oesophagus are accepted, reorientation of ideas about the pathology of the region is necessary. Some of the changes required will be indicated, and it is hoped to show that they allow the pathological observations to fall readily into a pattern which makes sense.

As already stated, cardiospasm, or achalasia of the cardia, involves the cardia.

Carcinoma of the cardia should be renamed carcinoma of the junctional epithelium or, simply, junctional carcinoma and should be regarded as a variety of oesophageal carcinoma.

The revolutionary change comes when reflux, peptic oesophagitis, and its complications are considered. In these cases junctional epithelium may be found extending for varying distances up the oesophagus, sometimes even as far as the level of the arch of the aorta. Many previous papers have referred to this state of affairs as "gastric" epithelium in the oesophagus (Barrett, 1950, 1952, 1957 ; Allison and Johnstone, 1953 ; Peters, 1955, 1958). In my opinion it can be accepted that "gastric" epithelium in the oesophagus is, in this type of case, almost always, if not always, of "cardiac" type, identical with what is here termed junctional oesophageal epithelium.

If it is agreed that this epithelium is oesophageal, all the cases of peptic oesophagitis previously described as having "gastric epithelium" in the oesophagus are merely cases in which the junctional epithelium extends further up than usual. Junctional epithelium that extends higher up the oesophagus than normal is not truly ectopic, in the sense of being tissue occurring in an organ in which it does not normally belong. Ectopic islands of gastric epithelium with oxyntic cells have been described as a rare finding in the oesophagus, but not in relation to the condition of peptic oesophagitis under discussion here.

It is interesting to follow the gradual change in outlook revealed in the literature over the past 10 years. Barrett (1950) defined the oesophagus as "the part of the foregut, distal to the crico- pharyngeal sphincter, which is lined by squamous epithelium." Since he regarded "cardiac" epithelium as gastric, this forced him to imply that oesophagus lined by this epithelium was not oesophagus but abnormal stomach, and in 1952 he published drawings showing "gastric" mucosa extending varying distances up the tube, in which he drew the epithelium of the whole stomach, fundus, and pylorus, as well as the lower part of the oesophagus, in the same black colour as if they were all the same. This oversimplification is permissible in a diagram, but it tended to make people imagine that the gastric epithelium might be digesting the oesophagus.

Allison and Johnstone (1953) corrected this impression by stressing the point that the columnar epithelium in the oesophagus in these cases is of cardiac type, which does not secrete digestive juices, and they advised that stomach mucosa with stomach musculature and peritoneum in the chest should be called thoracic stomach and oesophageal muscle with no peritoneum and stomach mucosa should be called oesophagus lined with gastric mucous membrane. They assumed without question that this "gastric" mucous membrane in the oesophagus was congenital. However, they noticed that it was nearly always associated with hiatal hernia, and wrote: "Such a congenital abnormality has been associated in the present series and in other reported specimens with a sliding hernia of the true stomach."

By 1957, Barrett's views had changed. He now referred to junctional epithelium in the oesophagus as "lower oesophagus lined by columnar epithelium," and stated that "it is neither true stomach nor oesophagus." He still regarded the condition as congenital and thought it was probably the result of failure of the embryonic lining of the gut to achieve normal maturity. Peters (1958) echoed this view, and stated that "the visceral attachment of the elastic hiatal ligament may be well below the squamo-glandular junction, and is then good evidence of congenital anomaly." He regarded the oesophagus lined by columnar epithelium as "an atypical structure, neither oesophagus nor stomach or, alternatively, both."

Moersch, Ellis, and McDonald (1959), in an excellent purely factual article, seem to be the first people to describe the changes following reflux oesophagitis without mentioning the word "congenital." This was a step forward. I wish to make the next step and declare that I do not believe that any convincing evidence has been advanced to show that any of the type of case described with more junctional epithelium than normal in the oesophagus is congenital. The only 
evidence that would convince me that the condition may sometimes be congenital would be the occasional finding of junctional epithelium extending an unusual distance up the oesophagus in stillborn babies. So far as I am aware, this observation has not been made. I am not impressed by Peters' (1958) series of 25 "congenital" cases of average age 62 and four over 80 years.

It is a wise principle in pathology never to regard anything as congenital until there is definite evidence that it was present at birth. It is too easy to label any tissue, which seems oddly placed or curiously developed and has no obvious cause, as congenital. This has happened over and over again. For instance, most of the conditions which 30 years age were called congenital cystic lung are now known to be acquired.

Medical reasoning about an abnormally long length of oesophagus lined by junctional epithelium (columnar) seems to have proceeded along the following lines: (1) The oesophagus is lined by squamous epithelium. (2) Oesophagus lined by columnar epithelium is not oesophagus. (3) Since columnar epithelium is not oesophageal, it must be gastric. (4) Therefore columnar epithelium in the oesophagus must be ectopic gastric epithelium. (5) As it is ectopic, it must be congenital. It would not matter if this reasoning remained on the theoretical plane without practical consequence, but this is not the case. There are two more steps in the reasoning. (6) Congenital anomalies are permanent. (7) There is only one thing to do with parts which are permanently abnormal, are causing trouble, and can be done without, and that is to cut them out.

Thus, the chain of reasoning, of which the first link is the belief that the oesophagus is lined only by squamous epithelium, ends with an indication for oesophageal resection. In a subsequent paper I hope to show that resections are usually unnecessary in these cases, even when the peptic oesophagitis has also led to a fibrous oesophageal stricture. When an apparently logical argument leads to action which, in practice, proves to have been needless, it is likely that something is wrong with the argument. In my opinion, it is the first step in the above reasoning which is at fault.

The following alternative reasoning, based on the belief that the lower centimetre or two of the oesophagus is normally lined by junctional epithelium which, though columnar, is truly oesophageal, is presented for consideration.

When the normal sphincteric and valvular mechanism in the lower oesophagus and oesophago-gastric junction, i.e., what I call the cardia, fails, usually as the result of the development of a sliding hiatal hernia, reflux from the stomach occurs and acid and pepsin reach the squamous epithelium and begin to digest it. In these patients the severity of the symptoms fluctuates. Long periods of remission may occur. Presumably, therefore, the amount of reflux or the digestive potency of the regurgitated stomach contents varies from time to time. In quiet periods some healing occurs, and in these periods the destroyed squamous epithelium may re-form, often with leucoplakia, or junctional epithelium, usually not very healthy-looking, may replace it. Where this occurs the area is given considerable protection from future reflux because normal junctional epithelium resists acid-peptic digestion.

Further reflux therefore attacks principally the squamous epithelium higher up. In the next remission it may be replaced by more junctional epithelium as a further protective reaction. With repetition over a long period the metaplastic junctional epithelium may creep higher and higher until it reaches the level of the arch of the aorta. It seldom extends higher than this.

At some time during the patient's illness a fibrous stricture may develop. It will form where the junction of squamous and columnar epithelium happens to be at the time, and may therefore be at any level up to the arch of the aorta. Subsequent reflux through the stricture may cause the columnar epithelium to climb above it. Subsequent abatement of oesophageal digestion may cause squamous epithelium to re-form below it. This type of stricture never forms at the oesophago-gastric junction. It is always at least a centimetre or two above it, because this is the lowest level to which the squamous epithelium ever extends.

This reasoning seems to fit the observed facts. In particular, it explains why islands of squamous epithelium are often found in the area newly lined by columnar epithelium and why submucous oesophageal glands frequently survive under it.

The last step in the reasoning is important. Since the abnormally highly situated columnar epithelium is probably an acquired metaplasia and not a permanent congenital abnormality, complete stoppage of the reflux (by efficient repair of the hiatal hernia) may cause it to retrogress and be replaced once more by squamous epithelium. The possibility of restoration towards normal greatly reduces the indication for resection.

\section{SUMMARY}

The oesophagus is defined as the tube conducting food from the throat to the stomach 
and all of this tube is regarded as oesophagus, irrespective of its lining.

The lower centimetre or two of this tube is normally lined by columnar epithelium, previously called cardiac and regarded as gastric, but in this paper called junctional and regarded as oesophageal. Thus the view that the oesophagus is a tube lined only by squamous epithelium is rejected.

The cardia is defined as the lower sphincteric part of the oesophagus between the attachment of the phreno-oesophageal ligament and the oesophago-gastric junction.

Some changes in opinions about lesions involving the lower end of the oesophagus, which must follow if these new views on its anatomy are accepted, are indicated. In particular it is pointed out that all the literature about gastric epithelium in the oesophagus in cases of reflux oesophagitis becomes invalid because with the new outlook it is simply oesophageal epithelium in the oesophagus.
It is probably neither ectopic, nor congenital, nor permanent, nor in need of resection but metaplastic and reversible.

I should like to thank Mr. N. R. Barrett for the elegant drawing on page 38 .

\section{REFERENCES}

Allison, P. R., and Johnstone, A. S. (1953). Thorax, 8, 87.

Barrett, N. R. (1950). Brit. J. Surg., 38, 175.

(1952). Proc. roy. Soc. Med., 45, 279

- (1957). Surgery, 41, 881.

Botha, G. S. M. (1958). Brit. J. Surg., 45, 569.

Cunningham's Text-book of Anatomy (1951), 9th edition. Ed. Brash, J. C. Oxford University Press, London.

Ellis, F. G., Kauntze, R., and Trounce, J. R. (1960). Brit. J. Surg., 47, 466.

Hurst, A. F., and Rake, G. W. (1930). Quart. J. Med., 23, 491.

Johnston, T. B., Davies, D. V., and Davies, F. (1958). Gray's Anatomy, 32nd ed. Longmans, Green., London.

Lerche, W. (1950). The Esophagus and Pharynx in Action. Charles C. Thomas, Springfield, Illinois.

Lockhart, R. D., Hamilton, G. F., and Fyfe, F. W. (1959). Anatomy of the Human Body. Faber and Faber, London.

Moersch, R. N., Ellis, F. H., and McDonald, J. R. (1959). Surg. Gynec. Obstet., $108,476$.

Peters, P. M. (1955). Thorax, 10, 269
Surgical opinions on the phreno-oesophageal ligament in hiatal hernia repair vary from those who ignore it (e.g., Sweet, 1948 ; Harrington, 1955) to those who regard its careful suture to the diaphragm under the edge of the hiatus as important (e.g., Allison, 1951 ; Clarebrough and Connell, 1959). Others acknowledge its existence but despise it. For instance, Tanner (1955) states that, in cases of hiatal hernia, "the phrenooesophageal ligament ... becomes infiltrated with fat and so stretched and tenuous that it becomes useless as a means of repair of the hernia," and Marchand (1959) states that it is "attenuated and inconspicuous when a hiatal hernia is present."

I have never found the phreno-oesophageal ligament inconspicuous, or useless as a means of repair, in these cases, and I agree with Allison and others that anchoring it below the diaphragm is a most important step in the operation. This paper is written to stress this point and to show how such opposite opinions may have arisen.

\section{The Normal Anatomy of the Ligament}

The phreno-oesophageal ligament is a layer of fascia rich in elastic fibres which arises from the under surface of the oesophageal hiatus as a continuation of the subdiaphragmatic fascia. which in turn corresponds to the fascia transversalis of the abdominal wall. It extends upwards in the hiatus in a conical fashion to be inserted into the adventitia of the oesophagus along a line about $2 \mathrm{~cm}$. above the oesophago-gastric junction (at level A in Fig. 1). At this insertion its elastic fibres merge with those of the adventitia and extend both upwards and downwards for a centimetre or two. Elastic fibres also pass from the adventitia among the muscle bundles of the oesophageal wall as far as the submucosa. The attachment of the ligament to the oesophagus is therefore very firm indeed and appears to be designed to distribute any tension in it over a wide area of the oesophageal wall. The space, like a circular wedge, between the conical ligament laterally, the cylindrical oesophagus centrally, and the peritoneum below, is filled with extraperitoneal fat. This wedge of fat is constant and is not much affected by general emaciation.

The anatomical point to be stressed here is the site of attachment of the ligament to the oesophagus, about $2 \mathrm{~cm}$. above the oesophago-gastric junction, where the oesophageal tube suddenly widens out into the stomach pouch and where the peritoneum is reflected from the stomach to the diaphragm (level $B$ in Fig. 1). This observation was made in the last century (Laimer, 1883) and 